\title{
FLUX AND SPECTRAL VARIATIONS OF 1E 1740.7-2942 OVER THE YEARS 2003-2012
}

\author{
Manuel Castro*, Flavio D'Amico, Francisco Jablonski, João Braga \\ Instituto Nacional de Pesquisas Espaciais (INPE), São José dos Campos, São Paulo, Brazil \\ E-mail: manuel.castro@inpe.br
}

The black hole system 1E 1740.7-2942 is usually the brightest hard X-ray source (above $20 \mathrm{keV}$ ) near the Galactic Center, but presents some epochs of low emission (below the INTEGRAL detection limit, for example). In this work, we present the results of studies on 1E 1740.7-2942 over 10 years, using the instruments ISGRI/IBIS and JEM-X, both on board the INTEGRAL observatory. We fit the spectra with both the compTT and cutoffpl models. According to the fits and taking the mean value over the 10 years, we have obtained a plasma temperature in the range $\sim 20-90 \mathrm{keV}$, and an average powerlaw index of $1.41(\sigma=0.25)$. We have also made a LombScargle periodogram of the flux in the $50-20 \mathrm{keV}$ band and found two tentative periods at 2.90 and 3.99 days. We present here the preliminary results of this ongoing work.

10th INTEGRAL Workshop: "A Synergistic View of the High Energy Sky" - Integral2014, 15-19 September 2014

Annapolis, MD, USA

${ }^{*}$ Speaker. 


\section{Introduction}

The source 1E 1740.7-2942 is a black hole candidate nearby the center of our Galaxy. It was discovered by the Einstein satellite [1] and it was thoroughly studied by several high energy missions like SIGMA/GRANAT, INTEGRAL, Suzaku and Chandra [2, 3, 4, 5]. It was also the subject of a recent study by our group [6], where we modelled the soft-to-hard X-ray spectrum as due to (thermal) Comptonization.

The INTEGRAL satellite [7] has already provided an uniform observation database of 1E 1740.72942. This database is suitable for a study of any possible long-term variability of the source. One interesting goal of such a study is the search for modulations in the X-ray lightcurve to reveal any possible orbital period. Reported orbital periods for 1E 1740.7-2942 [8,9] are still tentative, and a more precise determination of this period would be very important in the search for a (longer wavelength) counterpart of 1E 1740.7-2942, a challenging task that remains to be accomplished.

In this work we show the results of an ongoing effort to determine the orbital period of 1E 1740.7-2942 (if there is one). We have made use of the INTEGRAL database consisting of all of the 1E 1740.7-2942 observations from 2003 up to 2012. We have found two marginally significant tentative periods at $\sim 2$ and $\sim 4$ days. In the next sections we describe our database, our data reduction and analysis, and discuss our results.

\section{Data Reduction and Analysis}

From the public archive of INTEGRAL, we downloaded all of 1E 1740.7-2942 observations starting at 2003 up to 2012. 1E 1740.7-2942 spends most of its time in the so-called canonical low-hard state (LHS), where the fraction of the total flux is higher in the hard X-ray band than in the soft X-ray band. Accordingly, the source flux is below the sensitivity of JEM-X detectors in their energy range, being preferably monitored in the band covered by the IBIS telescope. As a result, Figure (1) shows how our retrieved 314 observations database is distributed between the 3 INTEGRAL imaging (unit) telescopes.

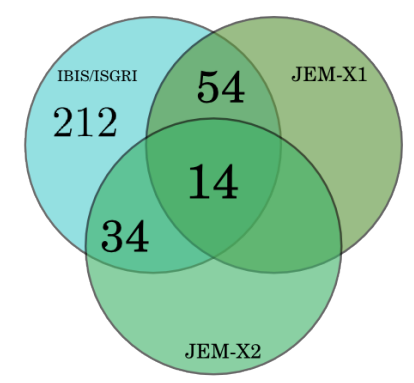

Figure 1: Our database of 314 retrieved observations of 1E 1740.7-2942. In the vast majority of cases (212) the source was imaged by IBIS alone. In 14 occasions the source was imaged by the 2 JEM-X units plus IBIS. In 54 with IBIS plus only the JEM-X1 unit and 34 with IBIS plus JEM-X2 unit.

Spectra were extracted for each individual revolution using an automatic extraction script based on the OSA 10.0 software. In some cases the spectra are built only with IBIS data, covering 
the $20-200 \mathrm{keV}$ energy band. When the source was also imaged by JEM-X, a spectrum from 5-200 $\mathrm{keV}$ could be extracted with the aid of our automatic script. After spectrum extraction, data were fitted also automatically with the aid of a Tcl script. For this preliminary study, we fitted the 20200 or the $5-200 \mathrm{keV}$ spectra (when present) with two models. The first one is a simple cutoffPL, suitable, for instance, to determine in which state the source was observed [10]. The second model is compTT, following the procedures of our previous study of the source [6]. In 14 occasions, as is shown in Figure (1), the spectrum extraction script needs to run three times (first run is always for IBIS), since both JEM-X units provided data. After fitting the data, our Tcl script saves the fit parameters (with error estimates) and calculated fluxes. We built our database with this kind of datafiles. In this first stage of our study, for simplicity, errors in the flux measurements are assumed to be within $\pm 5 \%$.

\section{Results}

The 1E 1740.7-2942 flux history of our database is shown in Figure (2). In Figure (3) we show the correlation between the fluxes in the two highest energy bands of this study. The histogram distributions for the 4 bands we adopted in this study are presented in Figure (4). The powerlaw index and plasma temperature data for our database are presented in Figures (5) and (6), respectively.

We have also produced a Lomb-Scargle periodogram using the R software environment [11]. This is shown in Figure (7).

\section{Discussion}

Our results have confirmed, as it is widely known, that 1E 1740.7-2942 spends most of its time in its canonical LHS, as derived from the powerlaw index of a fit with cutoffPL (see Figure 5). Spectral fitting in our database from 20 to $200 \mathrm{keV}$ or 5 to $200 \mathrm{keV}$ (when available) using compTT models, yields an average temperature of the electronic plasma, which (thermally) Comptonizes the soft seed photons, of $\sim 50 \mathrm{keV}$ (see Figure 6 ). The $\sim 300$ points in our database were helpful to better constrain this value. It must be emphasized, however, that at this point of our preliminary study the data for deriving $\Gamma$ and $\mathrm{kT}_{\mathrm{e}}$ are based on very low fluxes (see Figure 2) so the uncertainties are somewhat high.

As mentioned above, one of the main goals in this work is to search for possible binary periods. Our database is one of the best for such purposes, since 1E 1740.7-2942 spends most of its time in the LHS, i.e., the flux of 1E 1740.7-2942 is higher in the hard X-ray band ( $E>20 \mathrm{keV})$. Previous searches up to date where limited by databases in softer bands. INTEGRAL and the telescopes we used in this study, JEM-X and IBIS, are also imaging instruments and consequently 1E 1740.72942 flux is not affected by source confusion. Previous studies of searches for 1E 1740.7-2942 periods have made use (mostly) of data collected by non-imaging instruments and at the soft X-ray band $(E<20 \mathrm{keV})$.

Our results, which can be seen in (Figure 7), show the presence of periods at approximately 2.90 and 3.99 days. Even though relatively well above local noise level in the periodogram, the second period must be taken with caution since it is quite close to a multiple of the one-day sampling period used. In addition, the 2.90 -day period produces a folded light curve that is not 


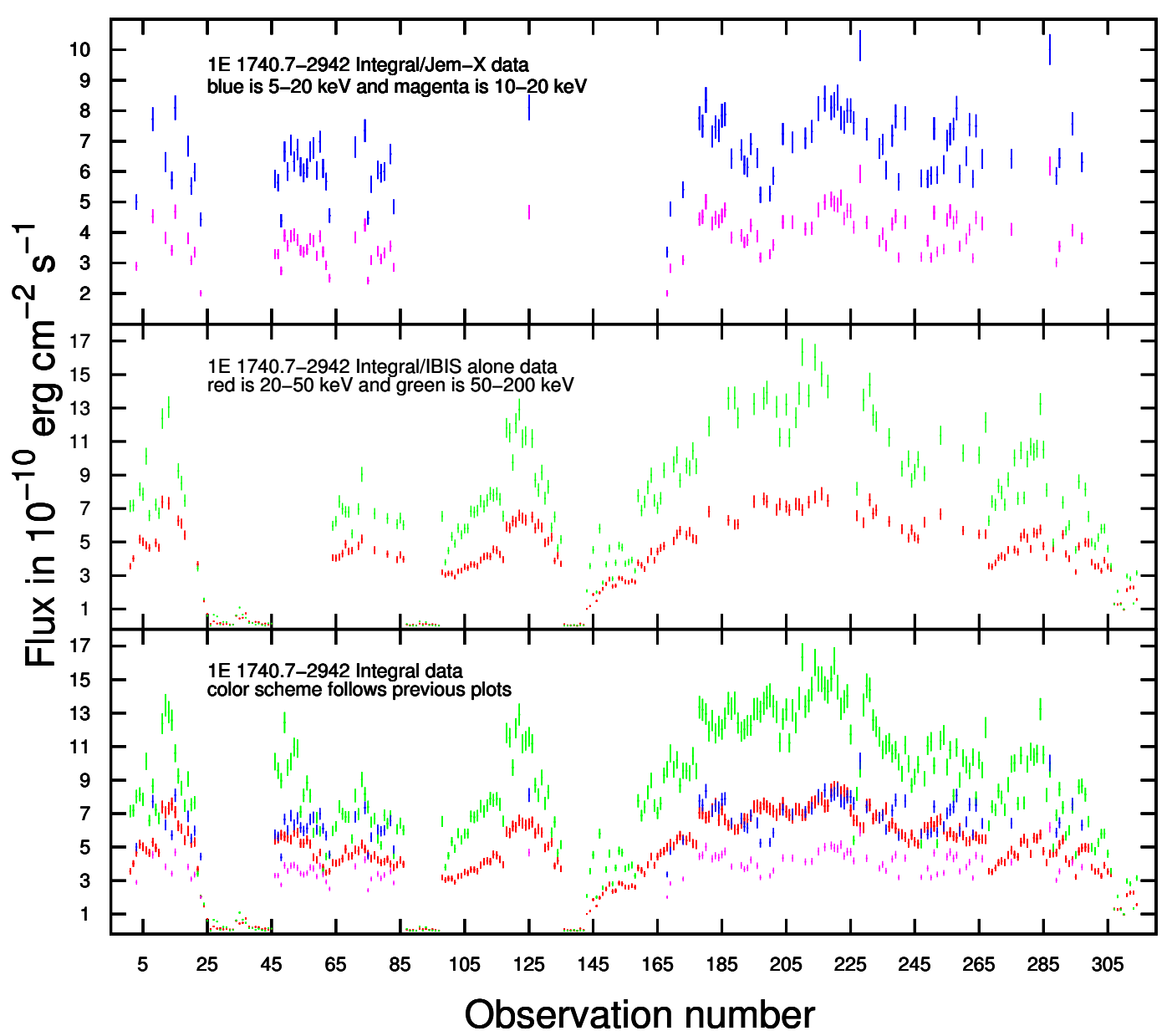

Figure 2: The flux history of 1E 1740.7-2942 based on our database.

very clean, which introduces uncertainties that we are still analysing. It must also be emphasized that much more sensitive studies using RXTE [9] have shown a period of $12.73 \pm$ days.

\section{Conclusion}

We have analysed data from the INTEGRAL mission to build flux and spectral history of 1E 1740.7-2942 from 2003 to 2012. Our data analysis is automatic and divided in two main fronts. First we use a script to automatically reduce the data using tasks of the OSA 10.0 software. Second, a Tcl script fits the data with XSPEC-aided commands. We collected, when possible, flux in 4 energy bands. Our data show, based on our fits with cutoffPL, that the source is observed to be in its canonical LHS state, where the flux in the hard X-ray band is greater than in the soft one. Our characterization of the 1E 1740.7-2942 spectra with compTT shows an average $\mathrm{kT}_{\mathrm{e}}$ of $50 \mathrm{keV}$. A Lomb-Scargle periodogram analysis has shown two tentative periods of 2.90 and 3.99 day. These results must be taken with caution due to uncertainties that are still being addressed. 


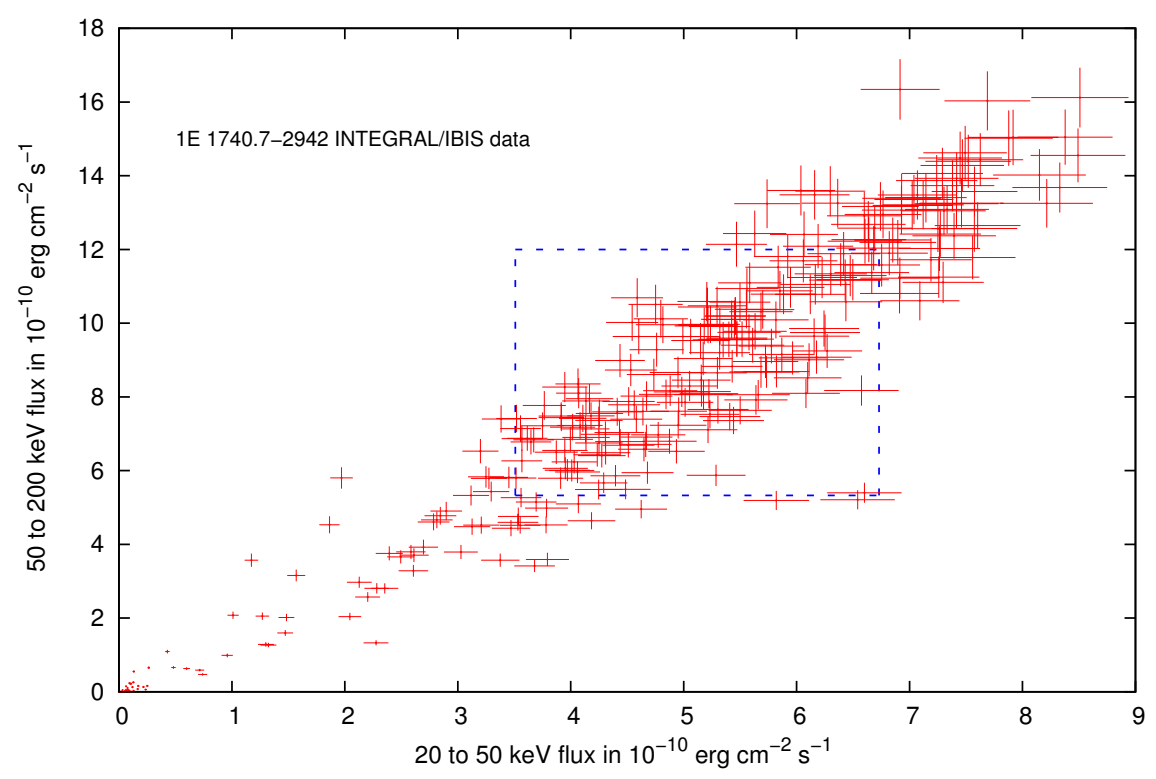

Figure 3: The fluxes in the two highest energy bands in this study. Shown in the plot also (in blue) is the region of average $\pm 1 \sigma$ for both bands. This shows the region (in flux) of preferred fluxes for the (canonical) high state where 1E 1740.7-2942 is commonly observed.

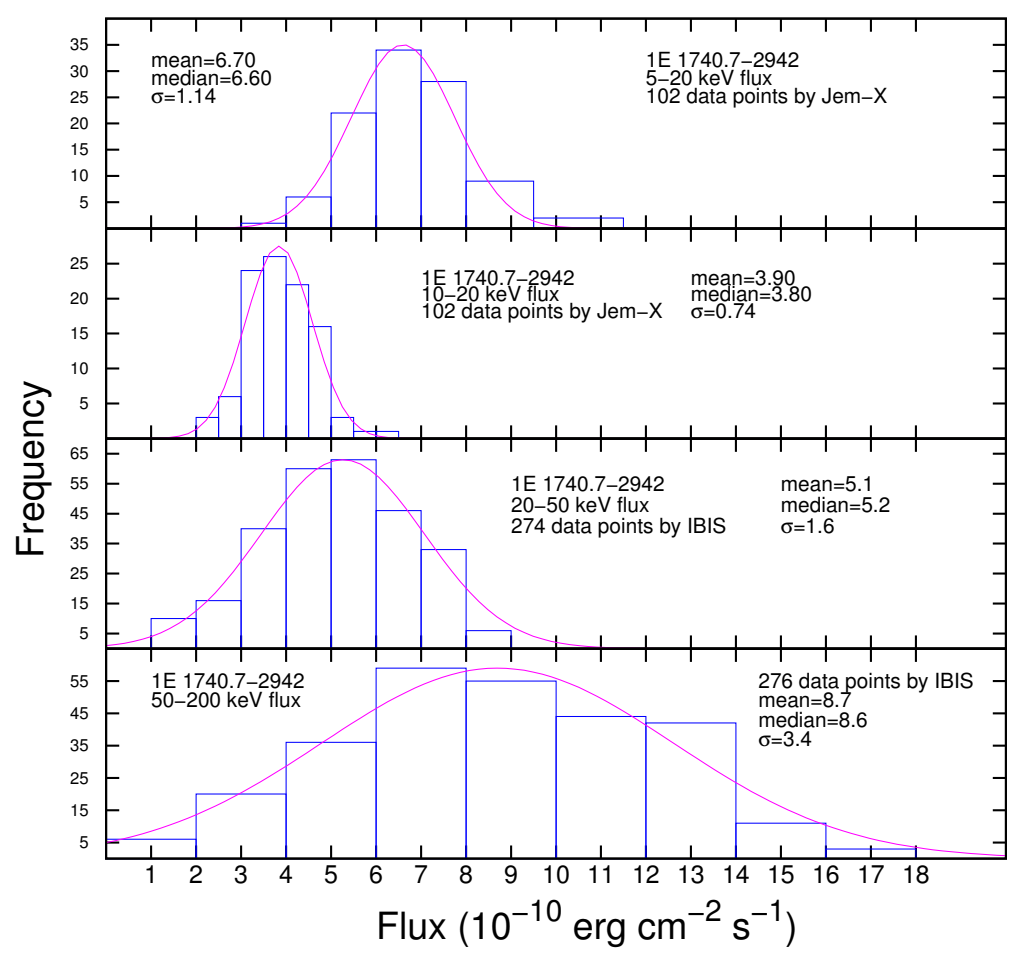

Figure 4: Histogram distribution for fluxes in the 4 energy bands considered in this study. 


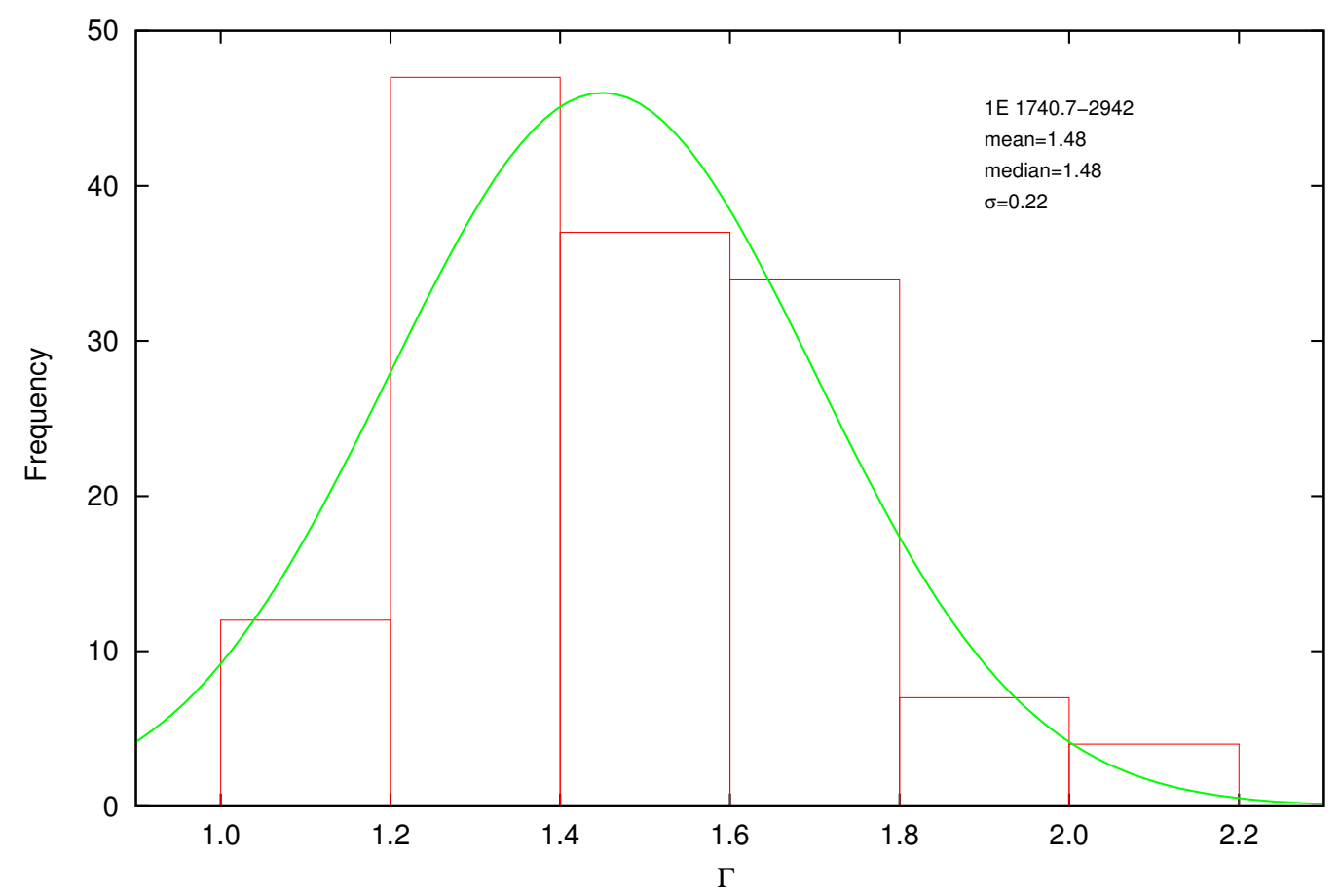

Figure 5: Histogram distribution for the powerlaw index $\Gamma$ derived from a cutoffPL fit in XSPEC.

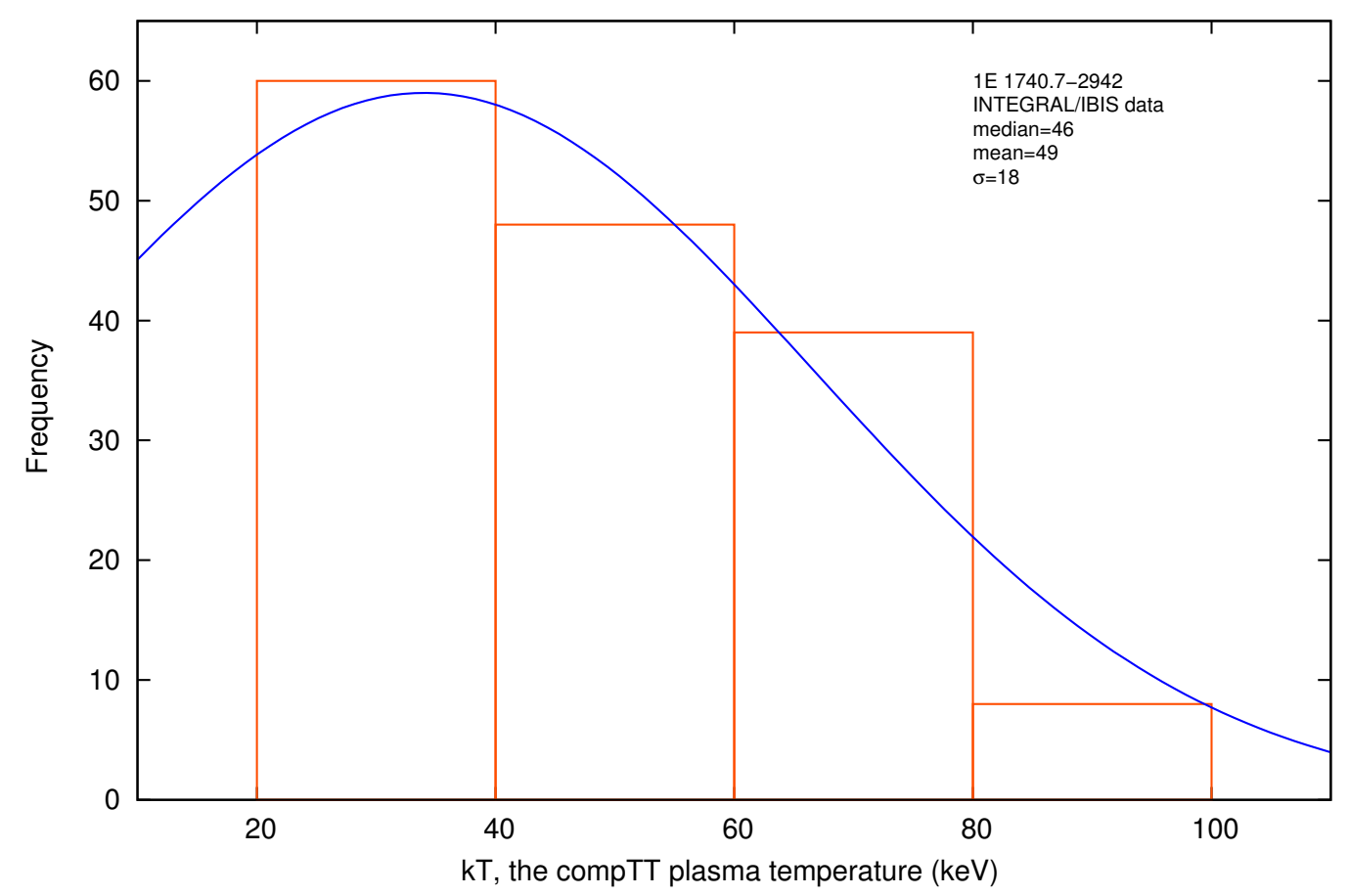

Figure 6: Histogram distribution for the plasma temperature kT derived from a compTT fit in XSPEC. 


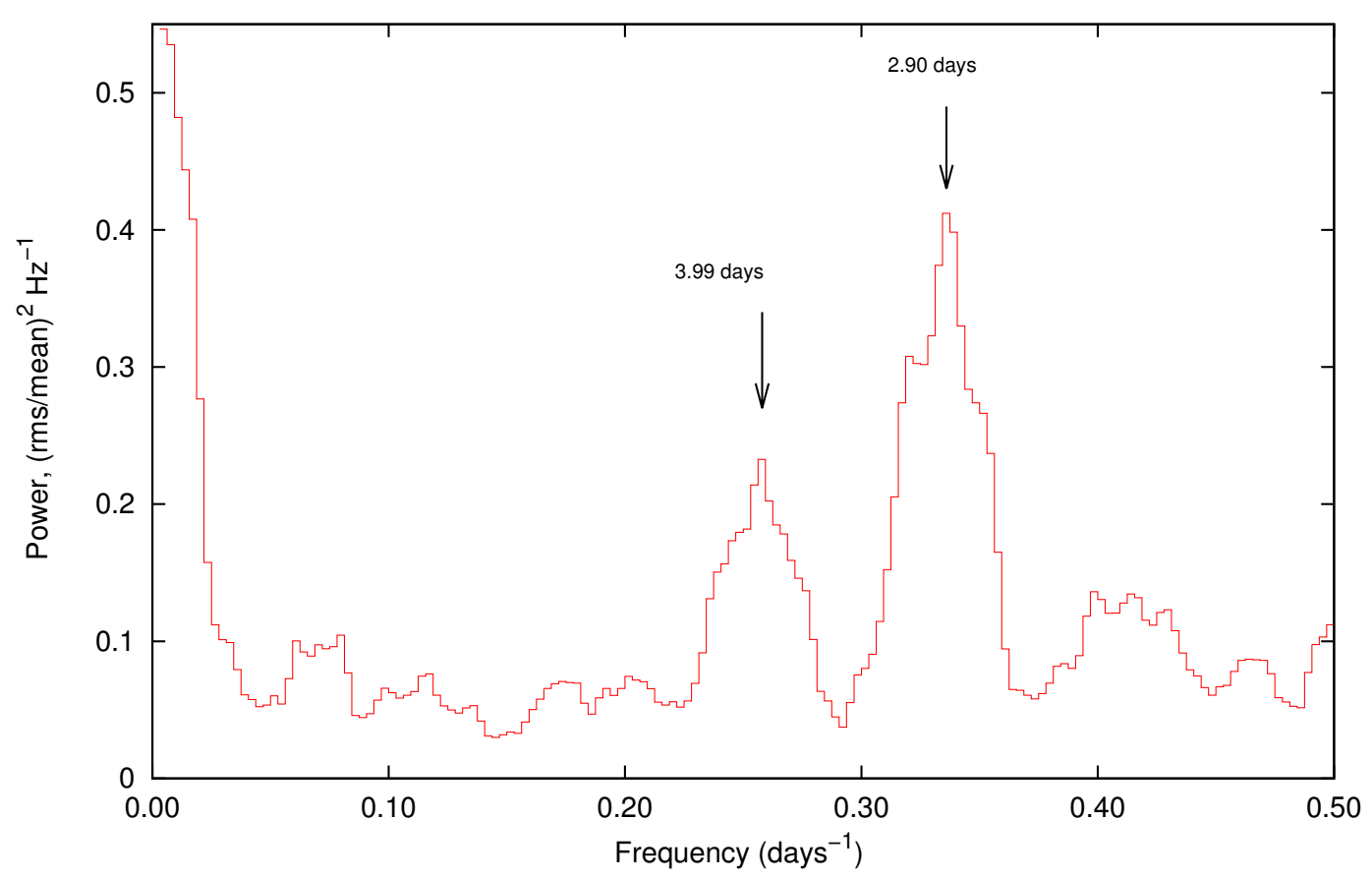

Figure 7: Lomb-Scargle periodogram of the $50-200 \mathrm{keV}$ flux showing two tentative possible periods for 1E 1740.7-2942 (see text for details).

\section{References}

[1] Hertz, P., Grindlay, J., The Einstein galactic plane survey - Statistical analysis of the complete X-ray sample,ApJ, 278, 137 (1984)

[2] Syunyaev, R., Gilfanov, M., Churazov, E., et al., Two Hard X-Ray Sources Near the Galactic Centerthe Known Source 1E 1740.7-2942 and the Newly Discovered Source GRS:1758-258, Sov. Astron. Lett+. 17. 50 (1991)

[3] Bouchet, L., del Santo, M., Jourdain, E., et al., Unveiling the High Energy Tail of 1E 1740.7-2942 With INTEGRAL, ApJ, 693, 1871 (2009)

[4] Reynolds, M., Miller, J., Suzaku Observations of the Galactic Center Microquasar 1E 1740.7-2942, ApJ, 716, 1431 (2010)

[5] Gallo, E., Fender, R., Chandra imaging spectroscopy of 1E 1740.7-2942, Mont. Not. R. Astron. Soc., 337, 869 (2002)

[6] Castro, M., D’Amico, F., Braga, J., et al., Confirming the thermal Comptonization model for black hole X-ray emission in the low-hard state, A\&A, 569, A82 (2014)

[7] Winkler, G., Courvoisier, T.J.-L., Di Cocco, G,. et al., The INTEGRAL mission, A\&A,411, L1 (2003)

[8] Smith, D., Heinfl, W., Swank, J., et al., X-Ray Timing in 1E 1740.7-2942 and GRS 1758-258, ApJ, 489, L51 (1997)

[9] Smith, D., Heindl, W., Swank, J., Orbital and Superorbital Periods of 1E 1740.7-2942 and GRS 1758-258, ApJ, 578, L129 (2002) 
[10] Remillard, R., McClintock, J., X-Ray Properties of Black-Hole Binaries, Annu. Rev. Astron. Astr., 44, 49 (2006)

[11] R Core Team, R: A Language and Environment for Statistical Computing, R Foundation for Statistical Computing, Vienna, Austria, 2008 